\title{
Otimização da produção de biodiesel a partir de óleo de coco babaçu com aquecimento por microondas
}

\author{
Ulisses Magalhães Nascimento*, Antônio C. S. Vasconcelos e Eduardo Bessa Azevedo \\ Núcleo de Biodiesel. Departamento de Química, Centro de Ciências Exatas e Tecnologia, Universidade Federal do Maranhão, CEP \\ 65080-040. São Luís - MA. \\ Fernando Carvalho Silva \\ Laboratório de Desenvolvimento de Tecnologias Ambientais. Instituto de Química de São Carlos, Universidade de São Paulo, CEP \\ 13560-970. São Carlos - SP.
}

\begin{abstract}
Resumo:As reações sob aquecimento por microondas geralmente apresentam significativa redução no tempo de reação e elevados rendimentos. Portanto, o objetivo desse trabalho foi adaptar um forno de microondas doméstico de forma adequada e segura para realização de reações de transesterificação, e otimizar o processo de produção de biodiesel por microondas usando óleo de coco babaçu como matéria prima. Para este fỉm, foi usado um planejamento composto central no qual se variou o tempo de irradiação, concentração de KOH e razão óleo:metanol. Análises estatísticas foram feitas para avaliarem a significância do modelo usado. As condições experimentais ótimas foram: razão óleo:metanol, 8,59, concentração de $\mathrm{KOH}, 2,19 \%$, tempo de irradiação, 70 segundos, dando rendimento de aproximadamente $100 \%$.
\end{abstract}

Palavras-chave: Biodiesel, Microondas, Transesterificação, Babaçu.

\section{Introdução}

O óleo de coco babaçu possui características interessantes para produção de biodiesel, considerando que a sua composição é predominantemente de ácido láurico. Este fato simplifica a reação para produção de biodiesel, pois esse ácido tem cadeia curta [1].

O Biodiesel é um biocombustível obtido a partir de óleos vegetais, gorduras animais ou óleos de frituras usados. Comumente é produzido pelo processo de transesterificação, no qual o óleo reage com um álcool de cadeia curta (metanol ou etanol), na presença de um catalisador homogêneo básico ou ácido. A transesterificação pode ser efetuada em batelada ou em fluxo contínuo utilizando aquecimento convencional. Outras metodologias para produção de biodiesel empregam fluidos su- percríticos, craqueamento térmico e aquecimento por microondas $[2,3,4]$.

$\mathrm{Na}$ produção de biodiesel sob aquecimento convencional, o calor é transferido ao meio reacional a partir da sua superfície por condução e convecção. Sendo assim, os métodos convencionais de aquecimento consomem mais energia e um tempo de produção de biodiesel na faixa de 5 a 30 minutos, para obter cerca de $90 \%$ de rendimento em ésteres $[5,6]$.

Há alguns anos tem-se observado o crescimento do interesse na aplicação de microondas para digestão de amostra para análises inorgânicas e biológicas. Além disso, tem havido esforços crescentes para aumentar a taxa de reação de sínteses orgânicas sob aquecimento por microondas [7].

Os reatores de microondas são muito úteis em laboratórios de Química, existindo no mercado 
uma grande variedade desses reatores para diversas finalidades, porém ainda com custos elevados, o que inviabiliza seu uso como equipamento de rotina em laboratório. Sendo assim, muitos pesquisadores têm optado por desenvolver projetos próprios de fabricação desses reatores ou utilizar fornos domésticos para fins laboratoriais, com ou sem adaptações [8].

O objetivo deste trabalho é adaptar um forno de microondas doméstico para reações de transesterificação e otimizar as condições do processo de produção de biodiesel a partir do óleo de coco babaçu com aquecimento por microondas.

\section{Parte Experimental}

\section{Adaptação do forno de microondas}

O forno de microondas utilizado em todos os experimentos foi um Panasonic, modelo NN-S65BK, 220 V, 60 Hz, 35 litros, com gerador de microondas na faixa de freqüência de 2.450 $\mathrm{MHz}$.

Como ilustra a Figura 1, para execução dos experimentos de transesterificação sob irradiação de microondas foi necessário adaptar um balão de vidro de duas bocas e um condensador de alta eficiência acoplados entre si por conectores de PTFE (politetrafluoretileno - Teflon ${ }^{\circledR}$ ), entre os quais foi inserida uma grade de aço inox 304 com pequenos furos de 1,0 mm de diâmetro, de forma a permitir a passagem de vapores e bloquear vazamentos de microondas. Além disso, foi inserido um termopar através de um furo de 3,0 $\mathrm{mm}$ de diâmetro até a segunda boca do balão. Para o controle de temperatura do forno de microondas, o termopar foi conectado a um controlador de temperatura com um adaptador eletrônico de acionamento das teclas liga e desliga de forma a automatizar o acionamento e interrupção da irradiação de microondas.

O prato giratório e seu motor de rotação foram retirados do forno. 


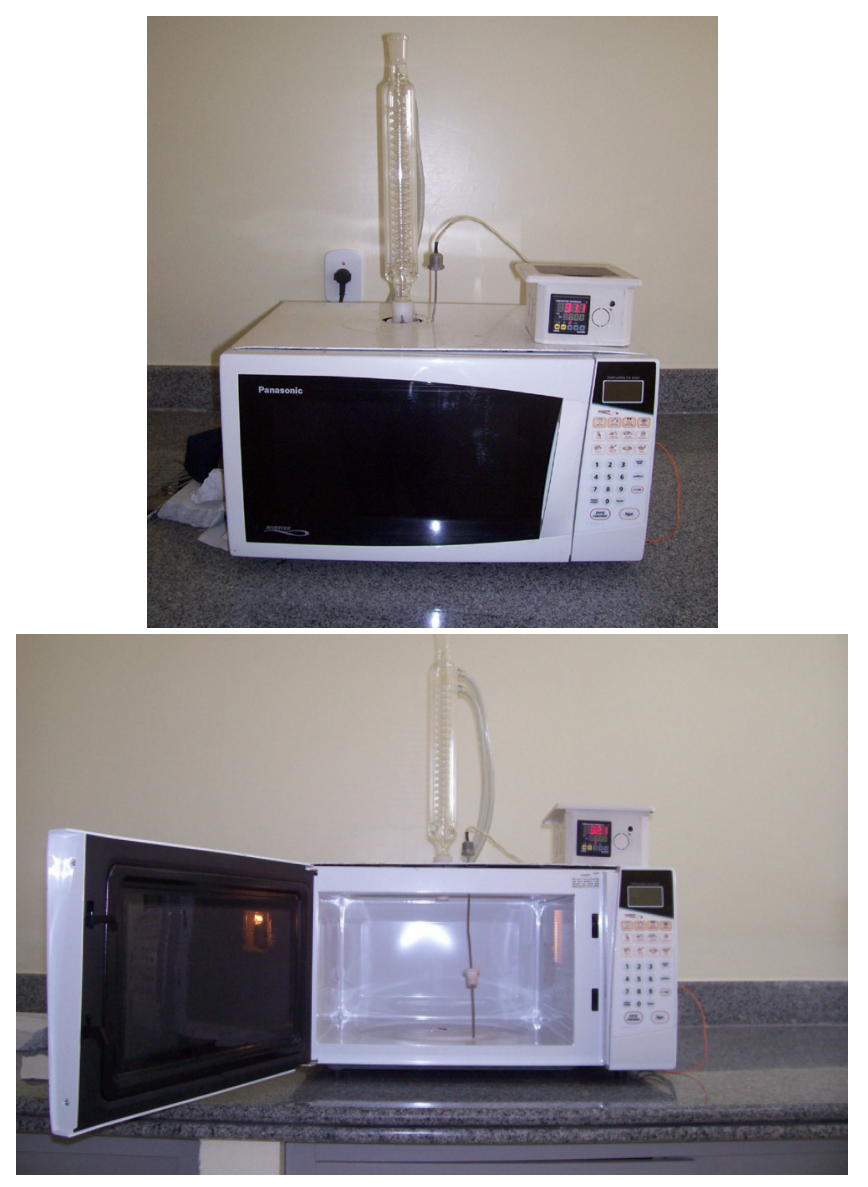

Figura 1 - (a) Forno de microondas doméstico adaptado para produção de biodiesel e (b) Parte interna do forno de microondasDeterminação da potência do forno

A determinação da potência do forno de microondas adaptado foi realizada indiretamente pela medida da temperatura da água destilada por um tempo estabelecido, contida em um erlenmeyer posicionado no centro da cavidade do forno, local onde posteriormente seria posicionado o balão com o meio reacional. $\mathrm{O}$ aquecimento foi realizado por 60,0 segundos, nas potências disponíveis no aparelho (P1 a P10). A potência (P) foi determinada pela razão entre a energia absorvida pela água $(n \cdot \overline{C P} \Delta T) \mathrm{e}$ o tempo de irradiação conforme a Equação 1, onde $n$ é a quantidade de matéria de água, neste caso 55,6 mols; $\overline{C P}$ é a capacidade calorífica molar da água $\left(75,3 \mathrm{~J} \mathrm{~K}^{-1} \mathrm{~mol}^{-1}\right) ; \Delta T$ é a variação de temperatura observada em K; e $t$ é o tempo de irradiação $(60 \mathrm{~s})$. Todos os experimentos foram feitos em triplicada e calculada a média aritmética [7]

$$
P=\frac{n \cdot \overline{C P} \Delta T}{t}
$$




\section{Análises físico-químicas do óleo de coco babaçu}

O óleo de coco babaçu utilizado nas reações de transesterificação foi obtido no comércio local e caracterizado seguindo metodologias normalizadas, segundo a Tabela 1.

Tabela 1. Propriedades e métodos para a especificação do óleo de babaçu.

\begin{tabular}{ll}
\hline \multicolumn{1}{c}{ Propriedades } & \multicolumn{1}{c}{ Métodos } \\
\hline Índice de Acidez $(\mathrm{mg} \mathrm{KOH} / \mathrm{g}$ óleo $)$ & SMAOFD $^{1} 2.201$ \\
Índice de Saponificação $(\mathrm{mg} \mathrm{KOH} / \mathrm{g})$ & SMAOFD 2.202 \\
Percentual dos ácidos graxos & SMAOFD 2.301 \\
Matéria insaponificável $(\%)$ & SMAOFD 2.401 \\
Índice de peróxido (meq $/ \mathrm{kg})$ & SMAOFD 2.501 \\
Umidade e matéria volátil $(\%)$ & SMAOFD 2.602 \\
Viscosidade Cinemática a $40^{\circ} \mathrm{C}\left(\mathrm{mm}^{2} / \mathrm{s}\right)$ & ASTM ${ }^{2}$ D 445 \\
Massa específica a $20^{\circ} \mathrm{C}\left(\mathrm{kg} / \mathrm{m}^{3}\right)$ & ASTM D 4052 \\
\hline
\end{tabular}

\section{Caracterização do biodiesel a partir do óleo de coco babaçu}

O biodiesel produzido em condições otimizadas foi submetido a alguns ensaios físico-químicos empregados no controle de qualidade do biodiesel, segundo a Tabela 2.

Tabela 2. Algumas propriedades e métodos para a especificação do Biodiesel.

\begin{tabular}{ll}
\hline \multicolumn{1}{c}{ Propriedades } & \multicolumn{1}{c}{ Métodos } \\
\hline Viscosidade Cinemática $\left(\mathrm{mm}^{2} / \mathrm{s}\right)$ a $40^{\circ} \mathrm{C}$ & ASTM D 445 \\
Massa específica a $20^{\circ} \mathrm{C}\left(\mathrm{kg} / \mathrm{m}^{3}\right)$ & ASTM D 4052 \\
Ponto de Fulgor $\left({ }^{\circ} \mathrm{C}\right)$ & ASTM D 93 \\
Enxofre Total $(\%$ massa $)$, máx. & ASTM D 4294 \\
Teor de Ésteres (\% massa), máx. & CEN* EN 14103 \\
\hline
\end{tabular}

*CEN - Comité Européen de Normalisation

\section{Análise cromatográfica}

Para a quantificação cromatográfica da conversão dos ácidos graxos do óleo de babaçu em ésteres metílicos, utilizou-se um cromatógrafo a gás, marca VARIAN, modelo CP-3800, acoplado a um detector de ionização em chama (CG-DIC). O cromatógrafo possui um injetor com divisão de fluxo (1:50) e uma coluna capilar de sílica fundida VARIAN (5\% fenil e 95\% dimetilpolisiloxano) com dimensões de $30 \mathrm{~m} \times 0,25 \mathrm{~mm} \times 0,25 \mu \mathrm{m}$. As seguintes condições cromatográficas foram utilizadas: $1,0 \mu \mathrm{L}$ de volume de injeção; hélio com $99,95 \%$ de pureza, como gás de arraste, com um fluxo de 1,2 
$\mathrm{mL} \mathrm{min}^{-1}$; temperaturas no detector e no injetor de 300 e $290^{\circ} \mathrm{C}$, respectivamente; programação de temperatura no forno de $150^{\circ} \mathrm{C}$ por $1 \mathrm{~min}$, rampa de 150 a $240^{\circ} \mathrm{C}$ a $10^{\circ} \mathrm{C} \mathrm{min}^{-1}$ e $240^{\circ} \mathrm{C}$ por $2 \mathrm{~min}$; rampa de 240 a $300^{\circ} \mathrm{C}$ a $15^{\circ} \mathrm{C} \mathrm{min}^{-1}$ e $300^{\circ} \mathrm{C}$ por 5 min. Inicialmente injetou-se um padrão de mistura de ésteres metílicos utilizando o mesmo método, para a identificação dos picos nos cromatogramas de cada amostra de biodiesel.

\section{Produção do biodiesel com aquecimento por mi- croondas}

O processo de produção de biodiesel metílico a partir de óleo de coco babaçu (BMB) consistiu nas etapas descritas pelo fluxograma apresentado na Figura 2.

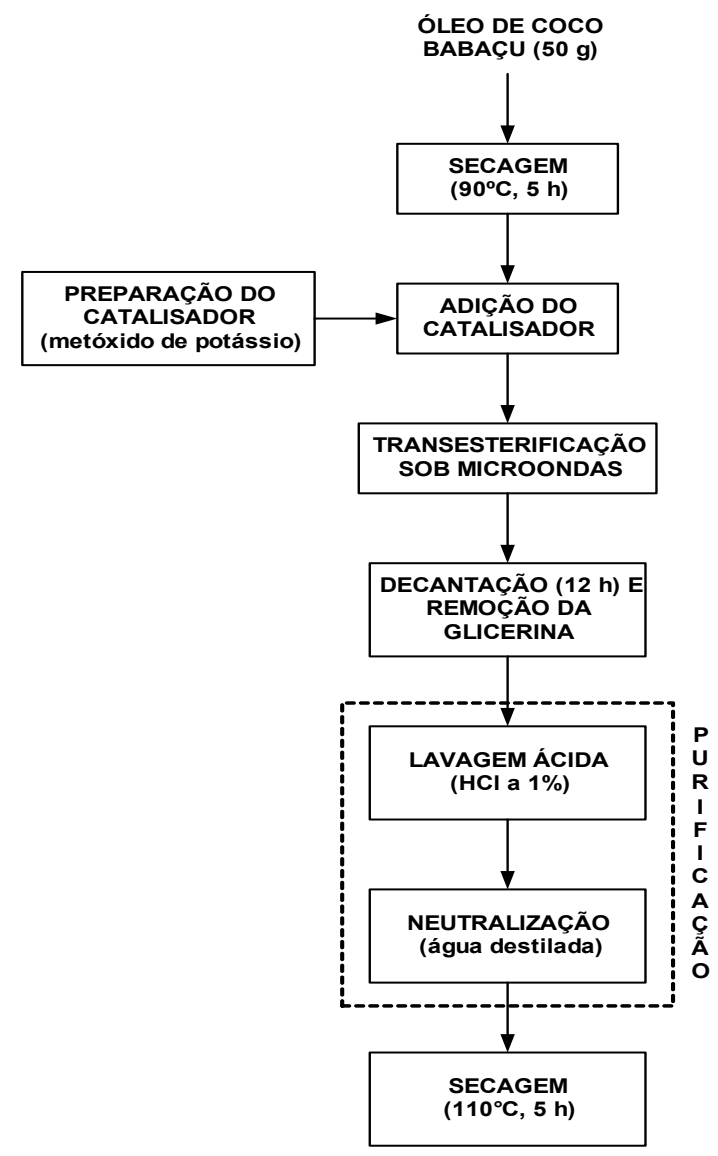

Figura 2 - Fluxograma do processo de produção do BMB.

\section{Planejamento experimental}

Para uma avaliação mais precisa da influência das variáveis sobre o rendimento da reação de transesterificação do óleo de coco babaçu, utilizou-se um planejamento composto central com três fatores, sendo os resultados avaliados através da aplicação de Metodologia de Superfície de Resposta (MSR) e Análises de Resíduos [9,10]. Para tratamento dos dados e obtenção dos gráficos estatísticos foi utilizado o programa Statistica ${ }^{\circledR}$ 7.0, utilizando-se o módulo de estimação não-linear para se obter uma equação empírica que representasse os dados experimentais obtidos. A partir dessa equação empírica, utilizou-se o pacote Solver do Excel $^{\circledR}$ para a obtenção do ponto de máximo rendimento em teor de ésteres.

Como apresentado na Tabela 3, as variáveis avaliadas foram: razão óleo/metanol, concentração de $\mathrm{KOH}$ e tempo de reação. A massa do óleo de coco babaçu, a potência e a temperatura usadas em todos os experimentos foram mantidos constantes em $50,0 \mathrm{~g}, 255 \mathrm{~W}(\mathrm{P} 9)$ e $60,0 \pm 10^{\circ} \mathrm{C}$, respectivamente. 
Tabela 3. Planejamento Composto Central realizado.

\begin{tabular}{lccccc}
\hline & \multicolumn{5}{c}{ Níveis } \\
\hline Variáveis & $\mathbf{- 1 , 6 8}$ & $\mathbf{- 1}$ & $\mathbf{0}$ & $\mathbf{+ 1}$ & $\mathbf{+ 1 , 6 8}$ \\
\hline Razão óleo:MeOH & $1: 3,6$ & $1: 5$ & $1: 7$ & $1: 9$ & $1: 10,4$ \\
Teor de KOH (\%) & 0,66 & 1,0 & 1,5 & 2,0 & 2,34 \\
Tempo de reação (s) & 26,4 & 40 & 60 & 80 & 93,6 \\
\hline
\end{tabular}

\section{Resultados e discussão}

\section{Adaptação do forno de microondas doméstico}

O condensador de alta eficiência adaptado ao forno para evitar a perda de álcool por evaporação, e os furos feitos com diâmetros de 1,0 $\mathrm{mm}$, menores que o comprimento de onda mínimo possível para as microondas $[11,12]$, foram eficazes, não tendo sido detectado em nenhum dos experimentos o vazamento de álcool e nem de microondas.

O controlador de temperatura com o adaptador eletrônico apresentou um bom funcionamento na faixa de temperatura utilizada.

Não foi adaptado nenhum tipo de sistema de agitação no forno, porque foi percebido em testes pré-liminares que seria possível fazer as reações sem necessidade de agitação.

A determinação da potência no forno mostrou que, nos diferentes níveis (P1 a P10), ela permanece praticamente a mesma, sendo o nível de potência P9 (255 W de potência, em média) o que mostrou o menor desvio-padrão.

\section{Caracterização do óleo de coco babaçu}

\section{Análises físico-químicas}

Embora não exista uma especificação oficial para os óleos vegetais utilizados na produção de biodiesel, estudos revelaram que elevados índices de acidez e umidade, por exemplo, reduzem o rendimento da reação [3]. Os resultados obtidos e mostrados na Tabela 4 apresentaram um índice de acidez e um teor de umidade dentro das faixas consideradas aceitáveis para produção de biodie- sel: 2,00 mg de $\mathrm{KOH}$ por grama de óleo e 0,50\%, respectivamente [13].

$\mathrm{O}$ índice de acidez indica a quantidade em massa de hidróxido de potássio necessária para neutralizar os ácidos graxos livres (AGL) nãoesterificados. Em meio básico, os AGL formam os sais de ácidos graxos (sabões) responsáveis pela formação de emulsão durante a lavagem do biodiesel, reduzindo assim o rendimento da reação [14]. O óleo de coco babaçu apresentou índice de peróxido de 1,14 meg $\mathrm{kg}^{-1}$, indicando uma baixa deterioração oxidativa, pois nos óleos este índice não deve ultrapassar o valor de $10 \mathrm{meg} \mathrm{kg}^{-1} \mathrm{de}$ amostra [15]. A viscosidade aumenta com o comprimento das cadeias dos ácidos graxos dos triglicerídeos e diminui quando aumenta o número de insaturações [14]. A viscosidade relativamente alta dos óleos, superior à da água, deve-se às atrações intermoleculares das grandes cadeias dos ácidos graxos, que constituem os triglicerídeos. A Tabela 4 demonstra que a viscosidade do óleo de coco babaçu foi de $34,84 \mathrm{~mm}^{2} \mathrm{~s}^{-1}$ a $40^{\circ} \mathrm{C}$, característico de oleaginosa constituída principalmente de ácidos graxos saturados. 
Tabela 4. Análises físico-químicas do óleo de coco babaçu.

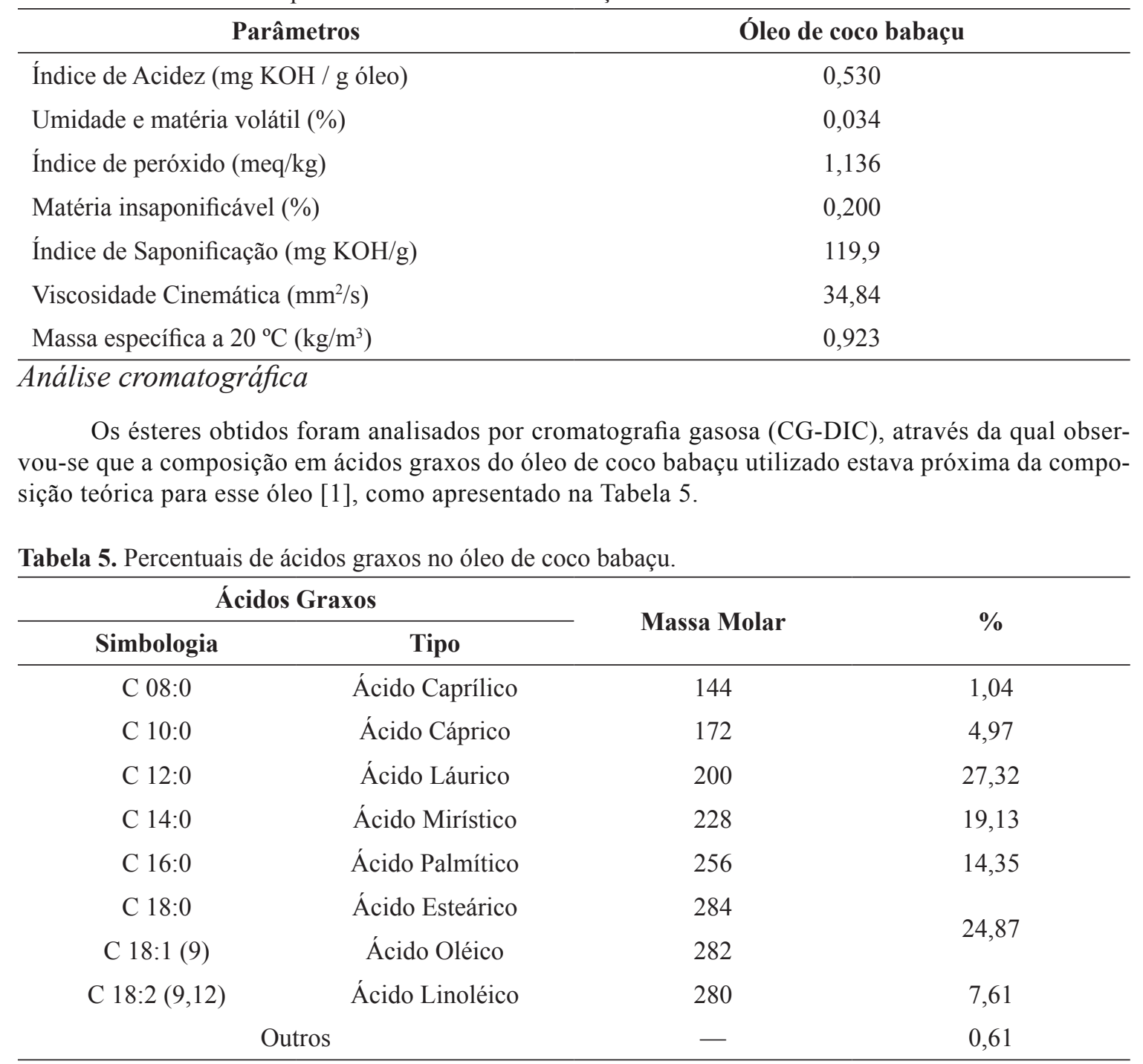

Planejamento Composto Central para a produção do biodiesel de óleo de coco babaçu

A matriz do planejamento experimental para o BMB foi o fatorial $2^{3}$ com 4 pontos centrais e 6 pontos axiais. Utilizaram-se como variáveis independentes a razão de quantidade de matéria óleo:metanol $\left(x_{1}\right)$, o teor de $\mathrm{KOH}\left(x_{2}\right)$ e o tempo de irradiação $\left(x_{3}\right)$; e como variável dependente o teor de ésteres, conforme apresentado na Tabela 6 .

A metodologia de produção de biodiesel a partir do óleo de coco babaçu aplicada nesse trabalho foi eficiente, e ainda, obteve-se uma considerável redução no tempo de reação, já que foram obtidos rendimentos acima de 90,0\% em teor de ésteres em tempos de irradiação menores que 30 segundos, enquanto que, em métodos que utilizam aquecimento convencional obtêm-se tempos de reação maiores que 5,0 minutos [5,6]. É possível que essa drástica redução no tempo de reação ocorra devido alta taxa 
de aquecimento, causada pela excelente capacidade do metanol em absorver as microondas irradiadas e converter essa energia em calor ( $\tan \partial=0,941)$, ou ainda, pela presença de íons no meio reacional proveniente do $\mathrm{KOH}$ que, poderão movimentar-se no meio por influência do campo elétrico, resultando na conversão da energia produzida pelo aumento na taxa de colisões em calor [12].

Tabela 6. Matriz do Planejamento realizado

\begin{tabular}{ccccc}
\hline \multirow{2}{*}{ Ensaios } & \multicolumn{3}{c}{ Variáveis dependentes } & (\%) \\
\cline { 2 - 4 } & $\boldsymbol{x}_{\mathbf{1}}$ & $\boldsymbol{x}_{\mathbf{2}}$ & $\boldsymbol{x}_{\mathbf{3}}$ & Ésteres \\
\hline 1 & -1 & -1 & -1 & 93,66 \\
2 & -1 & -1 & +1 & 92,27 \\
3 & -1 & +1 & -1 & 97,20 \\
4 & -1 & +1 & +1 & 98,22 \\
5 & +1 & -1 & -1 & 97,88 \\
6 & +1 & -1 & +1 & 94,53 \\
7 & +1 & +1 & -1 & 98,52 \\
8 & +1 & +1 & +1 & 98,79 \\
9 & $-1,68$ & 0 & 0 & 95,49 \\
10 & $+1,68$ & 0 & 0 & 96,19 \\
11 & 0 & $-1,68$ & 0 & 95,31 \\
12 & 0 & 1,68 & 0 & 99,07 \\
13 & 0 & 0 & $-1,68$ & 97,81 \\
14 & 0 & 0 & $+1,68$ & 97,53 \\
15 & 0 & 0 & 0 & 97,58 \\
16 & 0 & 0 & 0 & 98,03 \\
17 & 0 & 0 & 0 & 97,44 \\
18 & 0 & 08,31 \\
\hline
\end{tabular}

\section{Metodologia de Superfície de Resposta}

O Método da Superfície de Resposta (MSR) é uma técnica estatística muito útil para a modelagem e a análise de problemas nos quais as respostas são influenciadas por várias variáveis e que o objetivo é a otimização da resposta $[10,16]$. A partir do módulo de estimação não-linear, obteve-se um polinômio com o maior coeficiente de determinação possível que representasse os pontos experimentais, mostrado na Equação 2.

$$
\begin{gathered}
y=\beta_{0}+\beta_{1} x_{1}+\beta_{2} x_{2}+\beta_{3} x_{3}+\beta_{12} x_{1} x_{2}+\beta_{13} x_{1} x_{3}+\beta_{23} x_{2} x_{3}+\beta_{11} x_{1}^{2}+\beta_{22} x_{2}^{2}+\beta_{112} x_{1}^{2} x_{2}+ \\
+\beta_{221} x_{2}^{2} x_{1}+\beta_{112233} x_{1}^{2} x_{2}^{2} x_{3}^{2}
\end{gathered}
$$


A Figura 3 mostra o gráfico de contorno da interação das variáveis $\mathrm{KOH} \times$ óleo:MeOH, para um tempo de irradiação de $60 \mathrm{~s}$, onde a partir de uma avaliação isolada dessa interação observa-se uma ampla faixa em que pode-se obter rendimento de $100 \%$ em teor de ésteres, demonstrando uma boa robustez do processo de produção biodiesel testado.

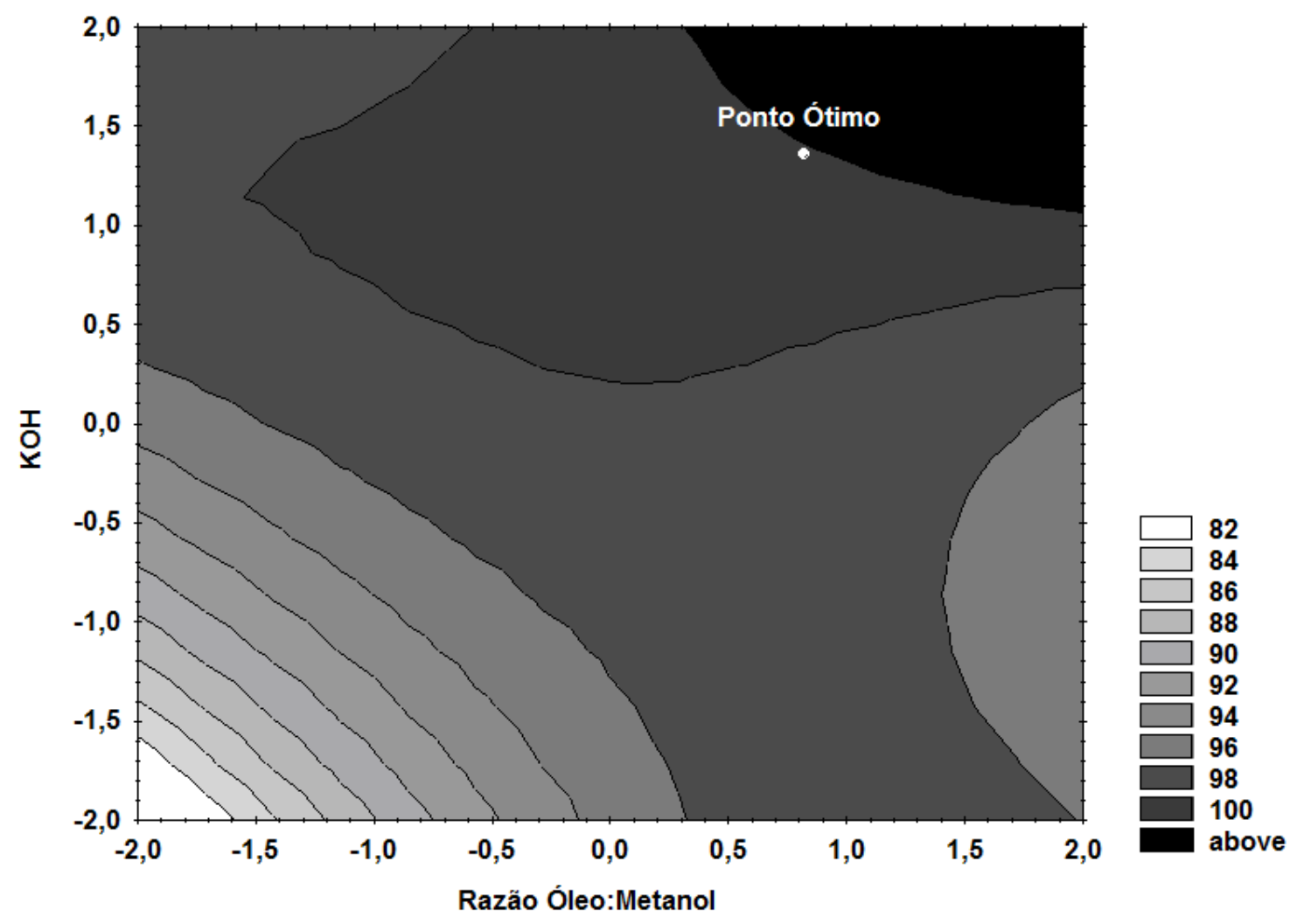

Figura 3 - Gráfico de contorno da interação $\mathrm{KOH} \times$ óleo:MeOH para o tempo de irradiação de $60 \mathrm{~s}$ (variáveis codificadas).

Ainda na Figura 3, mostra-se o ponto ótimo para a produção de BMB com aquecimento por microondas que foi estimado pelo Solver a partir da Equação 2: razão óleo/MeOH, 8,59; KOH, 2,19\%; e tempo, $70 \mathrm{~s}$, como pode ser percebido, todos os valores estão dentro da faixa estudada nesse trabalho o que denota um modelo robusto e preditivo.

\section{Análise de resíduos}

A análise de resíduos é imprescindível para avaliar o ajuste de qualquer modelo. Um modelo que deixe muitos resíduos é um modelo ruim. Em um modelo ideal não haveria resíduos, ou seja, os resultados observados seriam iguais aos previstos $[10,16]$. 
A Tabela 7 demonstra que todos os parâmetros utilizados no modelo não-linear, são estatisticamente significativos, pois nenhum destes tem um limite de confiança que o anule.

Tabela 7. Estimativa dos parâmetros para o modelo para o BMB.

\begin{tabular}{|c|c|c|}
\hline Parâmetros & Coeficientes & Limite de Conf. \\
\hline$\beta_{0}$ & 97,780 & $\pm 0,164$ \\
\hline$\beta_{1}$ & 0,208 & $\pm 0,169$ \\
\hline$\beta_{2}$ & 1,118 & $\pm 0,169$ \\
\hline$\beta_{3}$ & $-0,287$ & $\pm 0,109$ \\
\hline$\beta_{12}$ & $-0,574$ & $\pm 0,142$ \\
\hline$\beta_{13}$ & $-0,339$ & $\pm 0,142$ \\
\hline$\beta_{23}$ & 0,754 & $\pm 0,142$ \\
\hline$\beta_{11}$ & $-0,687$ & $\pm 0,116$ \\
\hline$\beta_{22}$ & $-0,210$ & $\pm 0,116$ \\
\hline$\beta_{112}$ & 0,681 & $\pm 0,221$ \\
\hline$\beta_{221}$ & 0,838 & $\pm 0,221$ \\
\hline$\beta_{112233}$ & $-0,503$ & $\pm 0,207$ \\
\hline
\end{tabular}

A partir da análise de variância (ANOVA) determinou-se o valor da estatística $\mathrm{F}$, que consiste na razão da média quadrática devido à regressão sobre a média quadrática devido ao erro, conforme a Tabela 8 .

Tabela 8. Análise de Variância do modelo para produção de Biodiesel a partir de óleo de coco babaçu com aquecimento por microondas.

\begin{tabular}{cccccc}
\hline Fonte de variação & S. Q. & G. L. & M. Q. & $\mathrm{F}_{\text {calc. }}$ & $\mathrm{F}_{\text {tab. }}$ \\
\hline Regressão & 60,40 & 11 & 5,49 & 13,54 & 4,03 \\
Resíduos & 1,11 & 6 & 0,18 & & \\
Falta de ajuste & 0,62 & 3 & 0,21 & 0,14 & 9,28 \\
Erro puro & 0,48 & 3 & 0,16 & & \\
Total & 61,51 & 17 & & \\
\% de variação explicada: & 0,982 & \\
\multicolumn{2}{c}{ \% máxima de variação explicável: }
\end{tabular}

A análise de regressão foi significativa, visto que o valor calculado de $F_{11,6}(13,54)$ é maior que o $\mathrm{F}_{\mathrm{R}, \mathrm{r}}$ tabelado $(4,03)$ e para a falta de ajuste, o valor calculado de $\mathrm{F}_{3,3}(0,14)$ foi menor que o $\mathrm{F}_{\text {fajep }}$ tabelado $(9,28)$, mostrando que o modelo é preditivo e significativo no nível de confiança de $95 \%$, além de não apresentar falta de ajuste dentro da faixa avaliada $[10,16,6]$. 
O ajuste do modelo também foi expresso pelo coeficiente de determinação $\left(\mathrm{R}^{2}\right)$, igual a 0,982 para o rendimento em teor de ésteres do BMB; demonstrando que esse modelo explica $98,2 \%$ dos $99,2 \%$ de variação explicável. Como o valor de $\mathrm{R}^{2}$ está próximo ao teórico, pode-se dizer que a modelagem mostrou-se adequada.

O gráfico do valor observado versus valor previsto pelo modelo é outra forma de se analisar a qualidade do ajuste do modelo estudado. A Figura 4 confirma que o modelo tem um bom ajuste, sendo que os valores previstos e observados são semelhantes, e ainda, que os resíduos estão normalmente distribuídos.

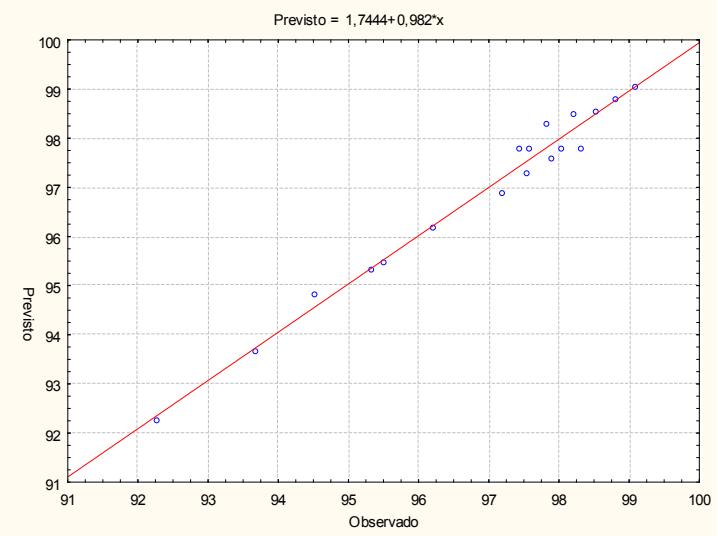

(a)

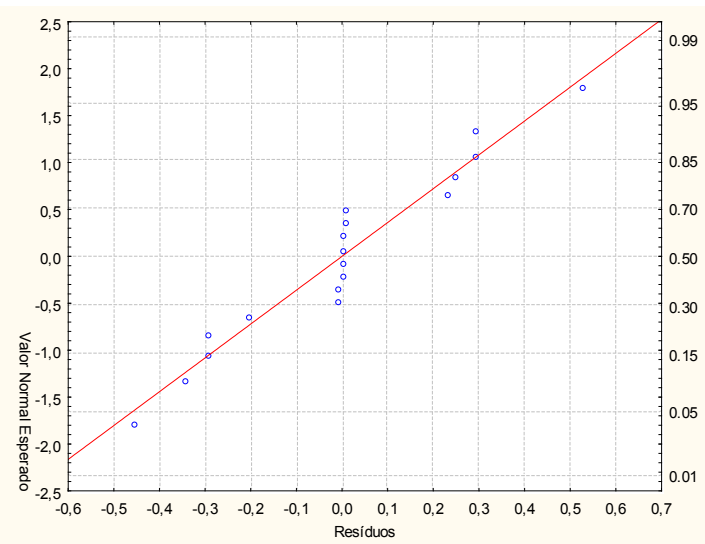

(b)

Figura 4 - Gráfico dos resíduos: valores previstos $\times$ valores observados; (b) Gráfico da probabilidade normal dos resíduos.Conclusão
As adaptações no forno de microondas para produção de biodiesel de óleo de coco babaçu foram realizadas com sucesso, funcionando de forma eficiente e segura. $O$ tempo de reação foi reduzido no mínimo em 10 vezes em relação aos métodos convencionais e sem detecção de vazamentos de irradiação de microondas.

O óleo de coco babaçu demonstrou ser uma boa alternativa como matéria-prima para produção de biodiesel, considerando que a qualidade desse óleo é adequada para a transesterificação e que foram obtidos elevados rendimentos de biodiesel dentro dos padrões de qualidade exigidos pelas legislações vigentes.

O modelo de regressão, no nível de $95 \%$ de confiança, mostrou que dos 99,2\% de variação explicável para o rendimento em teor de ésteres do BMB, foi possível explicar 98,2\% dessas variações com a equação empírica estimada.

O ponto ótimo para produção de BMB com aquecimento por microondas encontrado foi: razão óleo/MeOH, 8,59; KOH, 2,19\%; e tempo, 70 s.

\section{Agradecimentos}

Agradecemos a FAPEMA pelo financiamento da bolsa e ao CNPq pelo fomento desta pesquisa. 
ABSTRACT: Reactions under microwave heating present reduced reaction times and larger yields. Therefore, this work is aimed at adapting a domestic microwave oven and optimizing the transesterification reaction used in biodiesel production with microwave heating, using babaçu coconut oil as raw material. It was used a central composite design for varying irradiation time, $\mathrm{KOH}$ concentration, and oil:methanol ratio. Statistical analyses were performed in order to assess the significance of the model used. The optimized experimental conditions were: oil:methanol ratio, 8.59; $\mathrm{KOH}$ concentration, $2.19 \%$; and irradiation time, 70 seconds, giving an yield of approximately $100 \%$ regarding esters formation.

Keywords: Biodiesel, Microwave, Transesterification, Babaçu.

\section{Referências}

[1]. http://www.anvisa.gov.br/, acessada em agosto de 2007.

[2]. Lertsathapornuk, V.; Ruangying, P.; Pairintra, R.; Krisnangkura, K.; Chindaruksa, S.; Proceedings of the $1^{\text {st }}$ International conference on sustainable energy and green architecture, Bangkok, Thailand, 2003.

[3]. Ferrari, R. A.; Oliveira, V. da S.; Scabio, A.; Quim. Nova 2005, 28, 19.

[4]. Urioste, D.; Castro, M. B. A.; Biaggio, F. C.; de Castro, H. F.; Quim. Nova 2008, 31, 407.

[5]. Froehner, S.; Leithold, J.; Lima-Junior, L. F.; Quím. Nova 2007, 30, 2016.

[6]. de Moura, J. M. L. N.; Gonçalves, L. A. G.; Grimaldi, R.; Soares, M. da S.; Ribeiro, A. P. B.; Quim. Nova 2006, 29, 956 .

[7]. Jeyashoke, N.; Krisnangkura, K.; Chen, S.; J. Chromatography A 1998, 818, 133.

[8]. da Silva, F. de C.; Ferreira, V. F.; de Souza, M. C. B. V.; Quim. Nova 2006, 29, 376.

[9]. Rodrigues, M. I.; Iemma, A. F.; Planejamento de experimentos. $1^{\mathrm{a}}$ ed., Campinas - SP, 2005.

[10]. Neto, B. de B.; Scarminio, I. S.; Bruns, R. E.; Como fazer experimentos. $3^{\mathrm{a}}$ ed., Unicamp: Campinas-SP, 2007.

[11]. Sanseverino, A. M.; Quím. Nova 2002, 25, 660.

[12]. Lindstrom, P.; Tierney, J.; Wathey, B.; Westman, J.; Tetrahedron 2001, 57, 9225.

[13]. Canakci, M.; Gerpen, J.; Van, J.; Transactions of the ASAE 2001,44, 1429.

[14]. Moretto, E.; Fett, R.; Óleos e Gorduras vegetais - Na indústria de alimentos, Varela: São Paulo-SP, 1998.

[15]. Malacrida, C. R.; Braz. J. Food. Technol. 2003, 6, 245.

[16]. Montgomery, D. C.; Design and Analyses of Experiments, Wiley: New York, 2001. 